\title{
Aspirin resistance and diabetes mellitus
}

\author{
R. Ajjan • R. F. Storey • P. J. Grant
}

Published online: 16 January 2008

(C) Springer-Verlag 2007

Keywords Aspirin resistance - Clotting factors .

Diabetes mellitus $\cdot$ Platelets

\section{Abbreviations \\ COX-1 cyclooxygenase 1}

\section{Aspirin use in individuals with diabetes: where is the evidence?}

Despite multiple interventions to reduce the risk of cardiovascular disease, the majority of people with diabetes develop macrovascular complications, and mortality following myocardial infarction remains unacceptably high [1]. Antiplatelet agents are used for both the primary and secondary prevention of cardiovascular disease, although current guidelines are not consistent in their recommendation for the use of aspirin in diabetes [2]. In fact, there is little direct evidence supporting its efficacy in this group of patients. Instead, there is convincing data in the literature to suggest inadequate cardiovascular protection by aspirin in diabetes. In a meta-analysis of 287 randomised trials, antiplatelet treatment (aspirin in most studies) reduced the

\footnotetext{
R. Ajjan • P. J. Grant ( $\triangle)$

Academic Unit of Molecular Vascular Medicine,

The LIGHT Laboratories, University of Leeds,

Leeds LS2 9JT, UK

e-mail: p.j.grant@leeds.ac.uk

R. F. Storey

Cardiovascular Research Unit, University of Sheffield,

School of Medicine and Biomedical Sciences,

Sheffield, UK
}

risk of ischaemic events by $22 \%$, but the risk reduction in the subgroup with diabetes was only $7 \%$, which was not statistically significant [3]. This outcome was mirrored in the Primary Prevention Project trial, which reported that cardiovascular risk reduction with aspirin was marginal and non-significant in the presence of diabetes [4]. Despite this, there are no published studies specifically designed to evaluate the clinical efficacy of aspirin in individuals with diabetes, a surprising omission in the era of 'evidencebased' medicine.

These findings from clinical trials raise the question as to why there should be a reduction in the clinical efficacy of aspirin in patients with diabetes compared with a nondiabetic population. Diabetes is intrinsically associated with particular biochemical abnormalities that may have the capacity to diminish the effects of aspirin on platelet function and cardiovascular risk - a possibility that has led to the hotly debated concept of aspirin resistance $[5,6]$. Unfortunately, aspirin resistance suffers from a lack of a standardised definition, although now generally thought of as either (1) reflecting clinical aspirin resistance (or perhaps, more accurately, treatment failure), characterised by the occurrence of a thrombotic episode despite treatment with aspirin; or (2) biochemical aspirin resistance where platelet responses persist despite platelet exposure to aspirin. Controversy remains as to the cause of biochemical aspirin resistance, its relevance to clinical outcomes, and the place of aspirin treatment in the management of cardiovascular risk in diabetes patients. All of this highlights the urgent need to understand the mechanisms that underpin the interactions between diabetes and aspirin, to establish the role of aspirin in particular, and antiplatelet therapy in general, in the amelioration of cardiovascular events in individuals with diabetes. 


\section{Cardiovascular risk reduction: aspirin and its mode of action}

The activation of platelets is a complex process involving a number of steps (Fig. 1). The first step involves platelet adhesion to the site of vascular injury through interaction with the subendothelial extracellular matrix, including von Willebrand factor and collagen. Adhesion of platelets induces intracellular platelet signalling and activates integrins such as glycoprotein IIb/IIIa, which also interact with the extracellular matrix, thereby strengthening platelet adhesion. Once platelet adhesion is initiated, platelets produce a number of activating mediators, including thromboxane $\mathrm{A}_{2}$ and ADP, which together with activated glycoprotein IIb/ IIIa, recruit and activate more platelets to the site of injury. Thromboxane $\mathrm{A}_{2}$ is produced from arachidonic acid in a process mediated by cyclooxygenase 1 (COX-1). Thromboxane $\mathrm{A}_{2}$ plays a key role in mediating platelet activation through interaction with the thromboxane receptor, which can also be activated by prostaglandins $\mathrm{G}_{2}$ and $\mathrm{H}_{2}$. ADP, stored in platelets in the dense granules, activates platelets through the two protein G-coupled receptors $\mathrm{P}_{2} \mathrm{Y}_{1}$ and $\mathrm{P} 2 \mathrm{Y}_{12}$. Another important pathway in platelet activation is related to clotting factors. Thrombin is generated by the exposure of tissue factor in the vessel wall and subsequent activation of the clotting cascade. It acts as a key platelet activator through interaction with protease-activated platelet receptors. The antiplatelet agent aspirin inhibits COX-1, thereby limiting thromboxane $\mathrm{A}_{2}$ production (detailed below), whereas the active metabolite of clopidogrel blocks the $\mathrm{P}_{2} \mathrm{Y}_{12}$ receptor, thus inhibiting platelet activation.

Aspirin acts on platelets by irreversibly acetylating a serine residue in COX-1 in a reaction that is rapid and irreversible, so the effects endure for the life of the platelet ( $\sim 10$ days) [7, 8]. This inhibits the formation and release of the platelet agonist thromboxane $\mathrm{A}_{2}$ from activated platelets, such that aspirin effectively blocks the contribution of thromboxane $A_{2}$ to platelet aggregation and other platelet responses [9]. Thromboxane $\mathrm{A}_{2}$ has a role in thrombogenesis in animal models [10], and there is evidence of thromboxane generation within the coronary vascular bed in patients with coronary thrombosis [11]. Activation of phospholipase $\mathrm{A}_{2}$ and the liberation of arachidonic acid from membrane phospholipids is the first step in the pathway towards thromboxane $A_{2}$ release, and the activation of platelets by collagen switches on this pathway $[12,13]$. However, many other platelet agonists, such as ADP, serotonin and thromboxane $A_{2}$ itself, are linked to activation pathways that do not require collagen, and these do not lead to thromboxane $\mathrm{A}_{2}$ release [14]. This explains why aspirin has little or no effect on

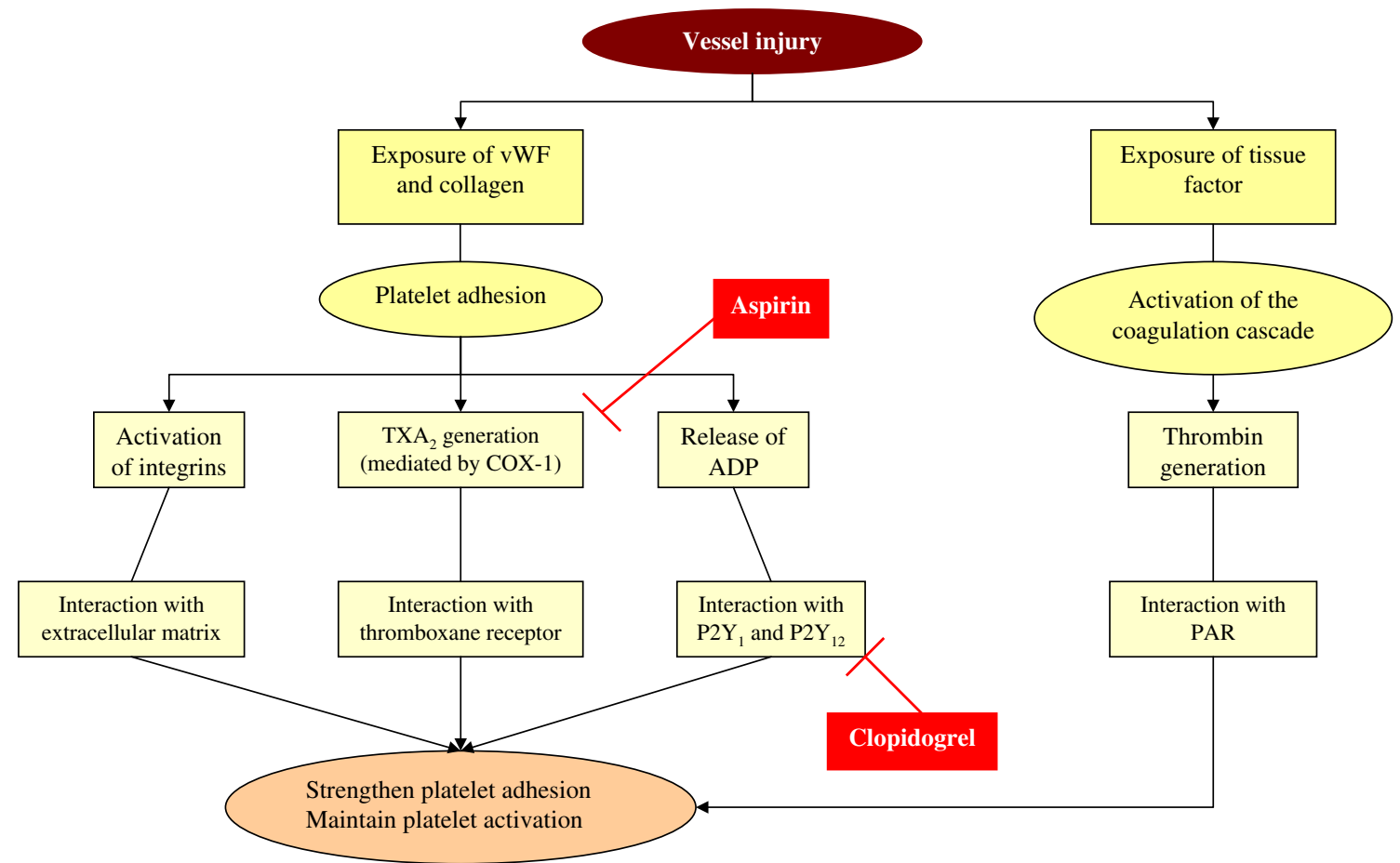

Fig. 1 Mechanisms of platelet activation. Following vessel injury, platelets interact with subendothelial extracellular matrix including von Willbrand factor (vWF) and collagen, resulting in platelet adhesion. Vessel injury also results in exposure of tissue factor and activation of the coagulation system, culminating in the production of thrombin. After adhesion, platelets become activated and produce integrins and thromboxane $\mathrm{A}_{2}$ and release stored $\mathrm{ADP}$, which collectively help to recruit and activate more platelets. Aspirin inhibits COX-1, thereby reducing thromboxane $\mathrm{A}_{2}$ generation, whereas the active metabolite of clopidogrel blocks the $\mathrm{P} 2 \mathrm{Y}_{12}$ receptor. PAR, protease-activated platelet receptor; $\mathrm{TXA}_{2}$, thromboxane $\mathrm{A}_{2}$ 
numerous aspects of platelet reactivity and why antiplatelet agents targeting other pathways may be necessary to prevent ischaemic cardiovascular events [15].

Another potential mode of action of aspirin is related to its effect on clotting factors and fibrin clot structure. Previous work has shown that fibrin clots composed of thin fibres, with small pores and a compact structure are associated with increased risk of thrombosis and cardiovascular disease [16], which may be due to slower clot lysis [17]. In vitro, clots formed from purified fibrinogen show increased fibrin gel porosity when incubated with aspirin, making them relatively less thrombotic [18]. In vivo, aspirin administration to healthy volunteers favourably alters fibrin clot structure - an effect that is more pronounced with lower doses of aspirin [19, 20]. Acetylation of fibrinogen is a likely mechanism for the observed in vitro and in vivo changes in clot structure after aspirin treatment [21]. Other mechanisms include modulation of thrombin generation and inhibition of coagulation factor XIII activation [22, 23].

\section{Assessment of biochemical aspirin resistance}

Importantly, recent work suggests that around $10-40 \%$ of people with diabetes display biochemical aspirin resistance $[24,25]$. However, this has been based on platelet function tests that assess aspects of platelet reactivity independent of thromboxane $\mathrm{A}_{2}$ release. Thus, these tests do not specifically measure how effectively aspirin has inhibited its target, COX-1. Given that baseline platelet reactivity is increased in diabetes (see below), this will give spuriously

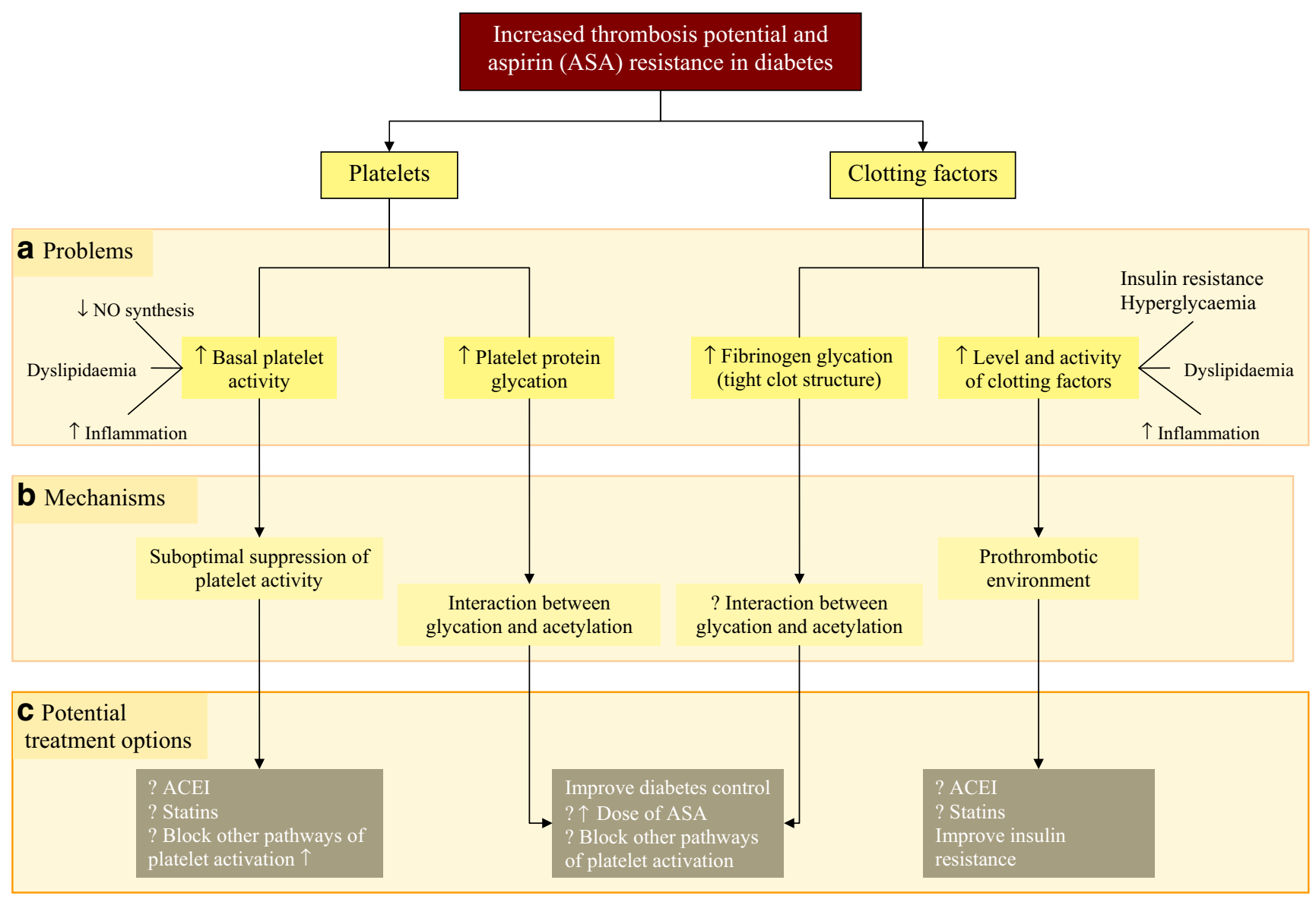

Fig. 2 Increased thrombotic risk and potential mechanisms of aspirin resistance in diabetes. There is an increase in basal platelet activity in diabetes, which may be responsible for suboptimal inhibition of platelet function by aspirin. Furthermore, increases in the plasma level and activity of clotting factors, associated with a prothrombotic clot structure, have been shown in individuals with diabetes. Therefore, reductions in platelet reactivity and the level and activity of coagulation factors by the use of statins, angiotensin converting enzyme inhibitors (ACEI) and modulation of insulin resistance, may improve the clinical efficacy of aspirin. Another mechanism for aspirin treatment failure is related to an increase in platelet and coagulation protein glycation, which may interfere with the acetylation process. Improved glycaemic control or the use of higher doses of aspirin may allow for more efficient acetylation of these proteins, consequently reducing the risk of thrombosis. NO, nitric oxide 
high figures for the prevalence of aspirin resistance. In support of this, when platelet thromboxane $A_{2}$ release is assessed by measurement of serum thromboxane $B_{2}$ levels, or the surrogate marker of arachidonic acid-induced platelet macroaggregation, it has been shown that poor platelet response to aspirin is rare [26-28]. Thus, many studies have reported relatively high rates of resistance because they have used methods that assess components of platelet reactivity that are independent of thromboxane formation and which are not expected to be inhibited by aspirin.

\section{Aspirin resistance/treatment failure in individuals with diabetes: potential mechanisms}

Although the concept of aspirin resistance in diabetes has been with us for more than 20 years [29], only a limited number of studies have investigated the potential mechanisms involved in this process (Fig. 2). First, there is an increase in platelet reactivity in patients with diabetes through decreased endothelial nitric oxide production, increased platelet turnover, altered platelet structure as a result of dyslipidaemia, and a disproportionate increase in intra-platelet calcium concentration [30]. Second, diabetes is characterised by an increased level and activity of prothrombotic clotting factors, associated with a tight clot structure and an impairment in fibrinolysis [31]. These effects on clotting factors are largely due to insulin resistance, dyslipidaemia and low-grade inflammation [30]. Third, a more diabetes-specific mechanism for increased aspirin resistance may be related to hyperglycaemia, particularly as no clear difference in biochemical aspirin resistance has been shown when comparing individuals with type 1 and type 2 diabetes [25]. An interaction between glycation and acetylation has been repeatedly shown [32-34], and increased glycation of platelet and coagulation factor proteins may interfere with the acetylation process to contribute to aspirin resistance in the presence of diabetes [35]. In vivo studies support this notion, as rats with streptozotocin-induced diabetes have a reduced platelet response to aspirin compared with nondiabetic animals, an effect related to reduced acetylation of platelet proteins [36]. If competition between acetylation and glycation of proteins affects the efficacy of aspirin in diabetes, it will be important to evaluate whether improving glycaemic control enhances the efficacy of aspirin and whether, in the presence of poor control, increased doses of aspirin are required [37, 38]. In support of this, a study comparing individuals with and without diabetes found no difference in biochemical aspirin resistance when higher doses of aspirin (325 mg/day) were used [39]. However, the use of $650 \mathrm{mg} /$ day of aspirin in the Early Treatment Diabetic Retinopathy Study (ETDRS) was associated with a non-significant reduction in vascular events in participants with diabetes, casting doubts about the efficacy of higher doses of aspirin [40]. Patients in this study were not taking agents that may improve the antithrombotic effects of aspirin, such as angiotensin receptor inhibitors or statins [41], and the general applicability of these results to current practice is uncertain.

\section{Conclusions}

Accumulating clinical and laboratory evidence suggest a reduced efficacy of aspirin in patients with diabetes. The exact mechanisms that underline the poor response to aspirin treatment in patients with diabetes are not entirely clear, but hyperglycaemia appears to be one factor involved. High blood glucose results in glycation of platelet proteins, making them less accessible to acetylation, potentially predisposing to treatment failure. Similar mechanisms may operate on clotting factors, which have been shown to undergo both glycation and acetylation. This interaction between acetylation and glycation may explain the greater effectiveness of clopidogrel in preventing vascular events in diabetes compared with low-dose aspirin [42]. Hypothetically, two simple approaches may help in increasing the efficacy of antithrombotic therapy in diabetes, including the administration of higher doses of aspirin or the use of other antiplatelet agents such as clopidogrel. The combination of aspirin and clopidogrel is also a possibility, particularly in those who show a partial response to either drug. Unfortunately, none of the above strategies can be recommended at present because of the lack of evidence supporting a clinical benefit of such an approach. It should be stressed that aspirin administration is associated with a significant risk of gastrointestinal haemorrhage, which seems to be dose dependent [43] and can be fatal. Therefore, aspirin-treated diabetes patients may be exposed to a considerable risk that may outweigh the small benefits of such treatment.

There is an urgent need for further clinical and basic research to clarify the prevalence of biochemical aspirin resistance in individuals with diabetes, to understand the relationship with clinical treatment failure and to elucidate the mechanisms involved. Establishing reliable indicators of efficacy will help to provide more effective and less hazardous treatment strategies in these individuals.

Acknowledgement R. Ajjan is funded by a Department of Health Clinician Scientist Award.

Duality of interest R. Ajjan has received consultancy fees/honoraria/educational grants from AstraZeneca, Sanofi-Aventis, GlaxoSmithKline, Daiichi Sankyo, Takeda, NovoNordisk, Merck Sharp \& Dohme and Pfizer. R. F. Storey has received grants/consultancy fees/ 
honoraria from: AstraZeneca, Eli-Lilly, Daiichi Sankyo, The Medicines Company. P. J. Grant has received consultancy fees/honoraria/ educational grants from GlaxoSmithKline, Eli-Lilly, Merck Sharp \& Dohme and Takeda.

\section{References}

1. Cubbon RM, Wheatcroft SB, Grant PJ et al (2007) Temporal trends in mortality of patients with diabetes mellitus suffering acute myocardial infarction: a comparison of over 3000 patients between 1995 and 2003. Eur Heart J 28:540-545

2. Nicolucci A, De Berardis G, Sacco M, Tognoni G (2007) AHA/ ADA vs ESC/EASD recommendations on aspirin as a primary prevention strategy in people with diabetes: how the same data generate divergent conclusions. Eur Heart J 28:1925-1927

3. Antithrombotic Trialists' Collaboration (2002) Collaborative meta-analysis of randomised trials of antiplatelet therapy for prevention of death, myocardial infarction, and stroke in high risk patients. BMJ 324:71-86

4. Sacco M, Pellegrini F, Roncaglioni MC, Avanzini F, Tognoni G, Nicolucci A (2003) Primary prevention of cardiovascular events with low-dose aspirin and vitamin $\mathrm{E}$ in type 2 diabetic patients: results of the Primary Prevention Project (PPP) trial. Diabetes Care 26:3264-3272

5. Mason PJ, Jacobs AK, Freedman JE (2005) Aspirin resistance and atherothrombotic disease. J Am Coll Cardiol 46:986-993

6. Sanderson S, Emery J, Baglin T, Kinmonth AL (2005) Narrative review: aspirin resistance and its clinical implications. Ann Intern Med 142:370-380

7. Roth GJ, Siok CJ (1978) Acetylation of the $\mathrm{NH}_{2}$-terminal serine of prostaglandin synthetase by aspirin. J Biol Chem 253:3782-3784

8. Patrono C, Ciabattoni G, Patrignani P et al (1985) Clinical pharmacology of platelet cyclooxygenase inhibition. Circulation 72:1177-1184

9. Kuster LJ, Frölich JC (1986) Platelet aggregation and thromboxane release induced by arachidonic acid, collagen, ADP and platelet-activating factor following low dose acetylsalicylic acid in man. Prostaglandins 32:415-423

10. Willerson JT, Golino P, Eidt J, Campbell WB, Buja LM (1989) Specific platelet mediators and unstable coronary artery lesions. Experimental evidence and potential clinical implications.. Circulation 80:198-205

11. Hirsh PD, Hillis LD, Campbell WB, Firth BG, Willerson JT (1981) Release of prostaglandins and thromboxane into the coronary circulation in patients with ischemic heart disease. N Engl J Med 304:685-691

12. Chang J, Musser JH, McGregor $H$ (1987) Phospholipase $A_{2}$ : function and pharmacological regulation. Biochem Pharmacol 36:2429-2436

13. Barnes MJ, Knight CG, Farndale RW (1998) The collagen-platelet interaction. Curr Opin Hematol 5:314-320

14. Lages B, Weiss HJ (1981) Dependence of human platelet functional responses on divalent cations: aggregation and secretion in heparin- and hirudin-anticoagulated platelet-rich plasma and the effects of chelating agents. Thromb Haemost 45:173-179

15. Storey RF (2006) Biology and pharmacology of the platelet $\mathrm{P} 2 \mathrm{Y}_{12}$ receptor. Curr Pharm Des 12:1255-1259

16. Fatah K, Silveira A, Tornvall P, Karpe F, Blomback M, Hamsten A (1996) Proneness to formation of tight and rigid fibrin gel structures in men with myocardial infarction at a young age. Thromb Haemost 76:535-540

17. Collet JP, Park D, Lesty C et al (2000) Influence of fibrin network conformation and fibrin fiber diameter on fibrinolysis speed: dynamic and structural approaches by confocal microscopy. Arterioscler Thromb Vasc Biol 20:1354-1361

18. He S, Blomback M, Yoo G, Sinha R, Henschen-Edman AH (2001) Modified clotting properties of fibrinogen in the presence of acetylsalicylic acid in a purified system. Ann N Y Acad Sci 936:531-535

19. Antovic A, Perneby C, Ekman GJ et al (2005) Marked increase of fibrin gel permeability with very low dose ASA treatment. Thromb Res 116:509-517

20. Williams S, Fatah K, Hjemdahl P, Blomback M (1998) Better increase in fibrin gel porosity by low dose than intermediate dose acetylsalicylic acid. Eur Heart J 19:1666-1672

21. Pinckard RN, Hawkins D, Farr RS (1968) In vitro acetylation of plasma proteins, enzymes and DNA by aspirin. Nature 219:68-69

22. Kessels H, Beguin S, Andree H, Hemker HC (1994) Measurement of thrombin generation in whole blood - the effect of heparin and aspirin. Thromb Haemost 72:78-83

23. Undas A, Sydor WJ, Brummel K, Musial J, Mann KG, Szczeklik A (2003) Aspirin alters the cardioprotective effects of the factor XIII Val34Leu polymorphism. Circulation 107:17-20

24. Fateh-Moghadam S, Plockinger U, Cabeza N et al (2005) Prevalence of aspirin resistance in patients with type 2 diabetes. Acta Diabetol 42:99-103

25. Mehta SS, Silver RJ, Aaronson A, Abrahamson M, Goldfine AB (2006) Comparison of aspirin resistance in type 1 versus type 2 diabetes mellitus. Am J Cardiol 97:567-570

26. Fontana P, Nolli S, Reber G, de Moerloose P (2006) Biological effects of aspirin and clopidogrel in a randomized cross-over study in 96 healthy volunteers. J Thromb Haemost 4:813-819

27. Schwartz KA, Schwartz DE, Ghosheh K, Reeves MJ, Barber K, DeFranco A (2005) Compliance as a critical consideration in patients who appear to be resistant to aspirin after healing of myocardial infarction. Am J Cardiol 95:973-975

28. Ohmori T, Yatomi Y, Nonaka T et al (2006) Aspirin resistance detected with aggregometry cannot be explained by cyclooxygenase activity: involvement of other signaling pathway(s) in cardiovascular events of aspirin-treated patients. J Thromb Haemost 4:1271-1278

29. DiMinno G, Silver MJ, Cerbone AM, Murphy S (1986) Trial of repeated low-dose aspirin in diabetic angiopathy. Blood 68:886-891

30. Ajjan R, Grant PJ (2006) Coagulation and atherothrombotic disease. Atherosclerosis 186:240-259

31. Dunn EJ, Philippou H, Ariens R, Grant PJ (2006) Molecular mechanisms involved in the resistance of fibrin to clot lysis by plasmin in subjects with type 2 diabetes mellitus. Diabetologia 49:1071-1080

32. Rendell M, Nierenberg J, Brannan C et al (1986) Inhibition of glycation of albumin and hemoglobin by acetylation in vitro and in vivo. J Lab Clin Med 108:286-293

33. Swamy-Mruthinti S, Carter AL (1999) Acetyl-L-carnitine decreases glycation of lens proteins: in vitro studies. Exp Eye Res 69:109-115

34. Watala C, Boncler M, Gresner P (2005) Blood platelet abnormalities and pharmacological modulation of platelet reactivity in patients with diabetes mellitus. Pharmacol Rep 57(Suppl):42-58

35. Watala C, Pluta J, Golanski J et al (2005) Increased protein glycation in diabetes mellitus is associated with decreased aspirinmediated protein acetylation and reduced sensitivity of blood platelets to aspirin. J Mol Med 83:148-158

36. Watala C, Ulicna O, Golanski J et al (2006) High glucose contributes to aspirin insensitivity in streptozotocin-diabetic rats: a multiparametric aggregation study. Blood Coagul Fibrinolysis 17:113-124

37. Watala C, Golanski J, Pluta J et al (2004) Reduced sensitivity of platelets from type 2 diabetic patients to acetylsalicylic acid (aspirin) its relation to metabolic control. Thromb Res 113:101-113

38. Takahashi S, Ushida M, Komine R et al (2007) Increased basal platelet activity, plasma adiponectin levels, and diabetes mellitus 
are associated with poor platelet responsiveness to in vitro effect of aspirin. Thromb Res 119:517-524

39. Gum PA, Kottke-Marchant K, Poggio ED et al (2001) Profile and prevalence of aspirin resistance in patients with cardiovascular disease. Am J Cardiol 88:230-235

40. Early Treatment Diabetic Retinopathy Study Investigators (1992) Aspirin effects on mortality and morbidity in patients with diabetes mellitus. ETDRS report 14. JAMA 268:1292-1300
41. Evangelista V, Totani L, Rotondo S et al (2005) Prevention of cardiovascular disease in type 2 diabetes: how to improve the clinical efficacy of aspirin. Thromb Haemost 93:8-16

42. Bhatt DL, Marso SP, Hirsch AT, Ringleb PA, Hacke W, Topol EJ (2002) Amplified benefit of clopidogrel versus aspirin in patients with diabetes mellitus. Am J Cardiol 90:625-628

43. Laine L (2006) Gastrointestinal bleeding with low dose aspirinwhat's the risk? Aliment Pharmacol Ther 24:897-908 